\title{
Advanced techniques for robotic polishing of aluminum mirrors
}

Hongyu Li, David Walker, Xiao Zheng, Guoyu Yu, Christina Reynolds, et al.

Hongyu Li, David Walker, Xiao Zheng, Guoyu Yu, Christina Reynolds, Wang Zhang, Tony Li, "Advanced techniques for robotic polishing of aluminum mirrors," Proc. SPIE 10692, Optical Fabrication, Testing, and Metrology VI, 106920N (15 June 2018); doi: 10.1117/12.2311625 


\title{
Advanced techniques for robotic polishing of aluminum mirrors
}

\author{
Hongyu Li ${ }^{* 1,4}$, David Walker ${ }^{1,2,3}$, Xiao Zheng ${ }^{1}$, Guoyu Yu ${ }^{1}$, Christina Reynolds ${ }^{1}$, Wang Zhang ${ }^{5}$ \\ and Tony $\mathrm{Li}^{1}$ \\ ${ }^{1}$ University of Huddersfield, at the National Facility for Ultra Precision Surfaces, OpTIC-Centre, St \\ Asaph, N. Wales, LL17 0JD, UK \\ ${ }^{2}$ University College London, Dpt. Physics and Astronomy, Gower St, London, WC1E 6BT, UK \\ ${ }^{3}$ Zeeko Ltd, Colville, Leicestershire, LE67 3WF, UK \\ ${ }^{4}$ Research Center for Space Optical Engineering, Harbin Institute of Technology, Harbin, 150001, \\ China \\ ${ }^{5}$ School of Mechanical Science and Engineering, Jilin University, Jilin, 130021, China
}

\begin{abstract}
Aluminum (pure or alloy) mirrors attract increasing interest, having Young's Modulus and density similar to glasses. Advantage of high diffusivity offsets disadvantage of high thermal expansion coefficient and means that the mirror reaches thermal equilibrium rapidly. High ductility supports extreme light-weighting and complex machining, including fluid-cooling channels in high-energy applications, and integral interface components. Aluminum mirrors are also tolerant to vibrations and shock loads. The material is amenable to single point diamond turning (SPDT) and does not require optical coating. However, SPDT tends to produce mid-spatial frequency artefacts, which are difficult to remove, especially for aspheres and free-forms. These introduce diffraction effects and compromise stray light performance.

In our previous research, we have demonstrated the potential of industrial robots to automate manual interventions with CNC polishing machines, and to provide surface-processing capabilities in their own right. We have also presented research concerning the mismatch between rigid and semi-rigid tools (including non-Newtonian tools), and aspheric surfaces. In this paper, we report on polishing of spherical and aspheric aluminum mirrors using an industrial robot. This includes tool-design, tool-path generation, texture control and removal of the mid-spatial frequency artefacts. We have investigated removal-rates and textures achieved, using different specialized slurries, polishing pads and special tool-paths. An effective process has been established, achieving Sa of $5 \mathrm{~nm}$ on a $400 \mathrm{~mm}$ square witness sample and a 490mm elliptical off-axis parabolic mirror.
\end{abstract}

Keywords: Aluminum, Mirror, Robotic polishing, Aspheric surface, Mid-spatial frequency

\section{INTRODUCTION}

Aluminum alloy, as a mirror material, has a high reflectivity over a wide spectral range from the infrared, through the visible, and into the ultraviolet. This makes it a good choice for many multispectral imaging applications [1,2]. Another reason for choosing this material is its high specific stiffness and good thermal properties [3]. In commercial and some space applications, low cost can be a significant driver. The use of aluminum permits direct single-point diamond turning of aspheres and free-forms (using tool-servoing). This side-steps the issues of significant sub-surface damage from hardgrinding of equivalent glass and ceramic substrates, with less time being needed to remove damage that does exist $[4,5]$. Typically, $5 \mathrm{~nm}$ Sa surface roughness of a diamond turned aluminum surface can be achieved, albeit with periodic diamond-turning features - well matched to infrared applications and many more-tolerant commercial applications at shorter wavelengths. To achieve low surface roughness, a nickel coating is sometimes applied to improve the ability to polish the mirror surface. One disadvantage of a nickel coating is that bi-metallic bending may occur, especially in cryogenic or high temperature applications, due to the disparity in thermal expansion coefficients [6]. Also, these coatings naturally compromise delivery times, and introduce some risk of delamination $[1,7]$.

*h.li@hud.ac.uk; phone: +44-1745535116

Optical Fabrication, Testing, and Metrology VI, edited by Sven Schröder, Roland Geyl, Proc. of SPIE

Vol. 10692, 106920N · @ 2018 SPIE · CCC code: 0277-786X/18/\$18 · doi: 10.1117/12.2311625 
SPDT tends to leave MSF (mid-spatial frequency) turning marks on the surface, which can compromise performance of functional surfaces, and be difficult to remove by post-processing (especially for aspheres and free-forms). Furthermore, for SPDT of larger or more massive parts, excitation of vibration-modes can exacerbate the problem. In optical application, these can produce diffractive side-lobes to images, and random scattered light, reducing detected signal-tonoise ratio. Wavelengths shorter than the infrared, and stray-light sensitive applications, often require surfaces superior to what SPDT alone can usually deliver [8]. As a general point, MSFs can also impact performance in non-optical applications. For the surfaces of turbine blades e.g. in titanium, MSFs may increase noise, reduced service-life and increased fuel-consumption, and the probability of detection in a military scenario. In bearing systems, including human joint implants, MSFs can increase wear and reduce implant lifetime.

Returning to aluminum, the logical approach to improving quality of an SPDT surface is a post-polishing finishing step. However, due to the softness and sensitivity to oxidization, aluminum is difficult to polish using traditional techniques. Our previous research has developed polishing processes to mitigate MSFs on glass and ceramic surfaces [9]. These and similar issues have led us to implement industrial robots both for process-automation, and as an intermediate processstep between $\mathrm{CNC}$ grinding of glassy materials and post-polishing [10]. In his paper, we now draw these threads together, and report on developing a new process for aluminum polishing using an industrial robot, which is amenable to future automated operation. Furthermore, we have deployed a non-Newtonian compliant tool [11] on an industrial robot (detailed in Section 4), to enhance capability on aspheric and freeform surfaces. We investigated the removal-rate and achieved texture of different specialized slurries, thereby establishing an effective two-step polishing process. The chain starts with a hard, flexible polishing pad on the non-Newtonian tool, used with a coarse \& fast slurry to remove any subsurface damage and MSF errors. It then finishes with a soft, flexible pad used with a refined \& slow slurry, to deliver the required surface texture. This process has been demonstrated on a nominally-flat $400 \mathrm{~mm} \times 400 \mathrm{~mm}$ aluminum plate, which was deformed into a freeform surface using a bending rig. This is introduced in following section.

\section{ALUMINIUM POLISHING TECHNIQUES INVESTIGATION}

In recent years, post-polishing has been applied in many applications to improve surface roughness and form of diamond turned surfaces - mainly plano optics and some aspheric surfaces. In practice, results have been limited by the material and slurry properties [8]. Due to the softness of aluminum, typical issues that arise in mechanical lapping and polishing, are smearing of the metal surface, and embedding of the abrasive material [1,12]. Using aqueous slurries of aluminum oxide abrasives, we have confirmed, the process is very sensitive to oxidization and scratching [13]. Following this, we investigated several specialized aluminum polishing slurries both on small spherical mirrors, and a 490mm diameter aspherical mirror, using Zeeko CNC polishing machines. Then, we optimized and applied these process on an industrial robot.

\subsection{Logitech CMP Solution on Zeeko IRP600 Precessions ${ }^{\text {TM }}$ polishing machine}

Logitech CMP (Chemical Mechanical Polishing) solutions have been developed for a wide range of semiconductor and opto-electronic polishing applications. Logitech CMP slurry SF1 is an alkaline colloidal-silica based slurry, which we have investigated on aluminum using the Zeeko IRP600 machine.

Zeeko Precessions ${ }^{\mathrm{TM}}$ polishing $[14,15]$ uses a compliant spherical tool (the 'bonnet') that is pressed against the surface to create a circular contact spot. The bonnet is rotated about its axis, and the rotation-axis precessed, to create a nearGaussian removal influence function. The tool contact-area (spot) and polishing-pressure can be modulated independently by changing respectively the axial position of the tool and its internal pressure. The 'Z-offset' (bonnet compression) defines the delivered spot-size for a specific size of bonnet. Different ranges of spot-size can be provided by exchanging bonnets between runs, and radii of curvature of bonnet from $20 \mathrm{~mm}$ to $320 \mathrm{~mm}$ are available. This gives the capability to optimize the process automatically using a limited range of different spot-sizes across a surface, and manually using different tools for different stages of the work, or for the different demands of smaller and large workpieces.

The part in this test was a 100mm diameter flat aluminum sample (6082 alloy). The surface of this part was milled and then smoothed by a hand lapping machine with C9 aluminum oxide abrasive. The polishing tool was a standard Zeeko inflated bonnet tool with radius of $80 \mathrm{~mm}$. The Logitech slurry SF1 was pumped onto the surface of the part, and recirculated by a second pump. The setup is shown in Figure1. 

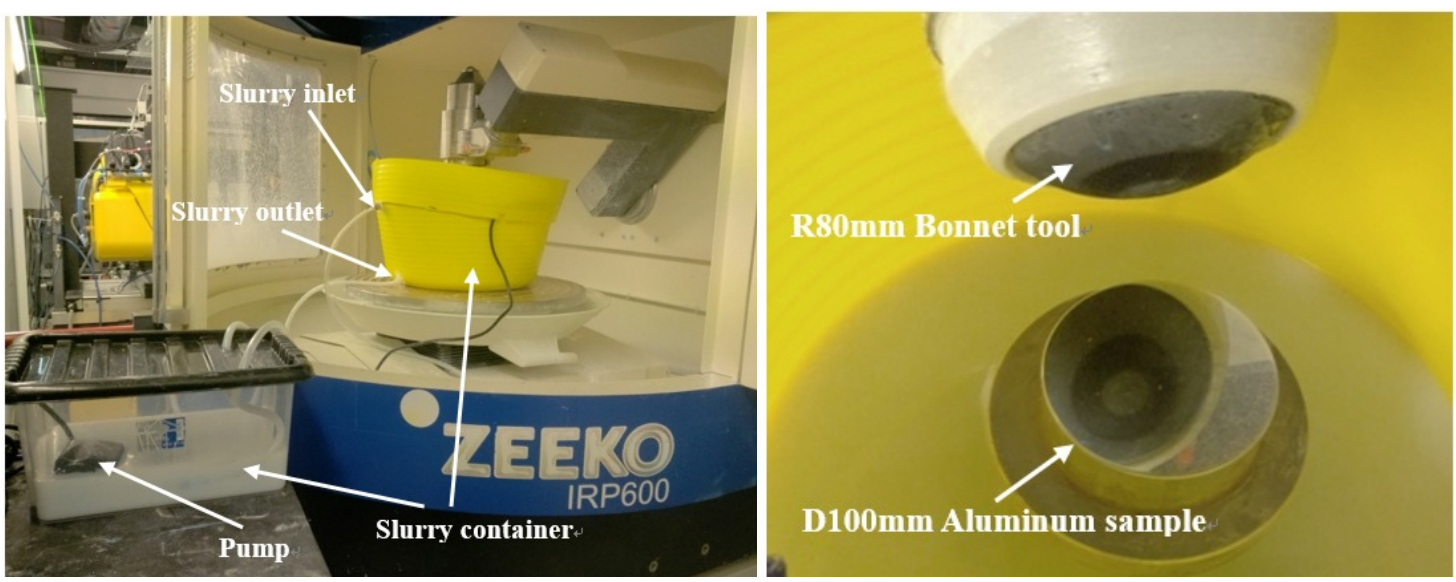

Figure 1. 100mm aluminum sample test set-up on Zeeko IRP600 machine

The test started with polyurethane cloth to achieve a fast removal-rate, with parameters as per Table 1. Two raster runs were performed perpendicularly to minimize the raster signature. The total polishing time was 22 minutes. The volumetric removal rate was tested using same processing parameters in Table 1 . The removal rate test was conducted by measuring the depth of a polishing trench, giving a volumetric removal rate of $0.557 \mathrm{~mm}^{3} / \mathrm{min}$. After 22 minutes polishing, the surface quality was as shown is Figure 2. Water marks and raster marks can be seen on the surface, due to the sensitivity of aluminum to oxidation, and softness of the material. Also crystallized hard particles were observed during polishing, which caused scratching. The hard polishing pad (3mm thickness polyurethane) with 1 bar bonnetpressure introduced 'orange-peel', due to the softness of aluminum. These effects led to degraded the surface texture, which was measured to be $\mathrm{Sa}=19.2 \mathrm{~nm}$ using a white-light interferometer, as shown in Figure 2.

Table 1 Process parameters using Polyurethane cloth

\begin{tabular}{|c|c|c|c|}
\hline Polishing Cloth & Polyurethane & Tool Overhang $(\mathrm{mm})$ & 0 \\
\hline Slurry & Logitech & Bonnet Pressure $(\mathrm{bar})$ & 1 \\
\hline Precess angle $(\mathrm{degs})$ & 15 & Raster-track Spacing $(\mathrm{mm})$ & 1 \\
\hline Head Speed $(\mathrm{rpm})$ & 1000 & Surface Feed $(\mathrm{mm} / \mathrm{min})$ & 500 \\
\hline Tool Offset $(\mathrm{mm})$ & 1 & Removal rate $\left(\mathrm{mm}^{3} / \mathrm{min}\right)$ & 0.557 \\
\hline
\end{tabular}

To address the slurry crystallizing and aluminum oxidizing, the polyurethane cloth was replaced by Uninap. Tool pressure was reduced to $0.5 \mathrm{bar}$, and offset to $0.5 \mathrm{~mm}$, as per Table 2, resulting in a measured volumetric removal rate of $0.483 \mathrm{~mm}^{3} / \mathrm{min}$. A modified procedure to use this slurry was advised by the supplier:

(1) At the end of a run, stop the slurry flow rate onto the pad while the process is still operating

(2) Then, flush the sample with deionized water for at least 20 seconds while the process is still running.

(3) Stop the process and remove the sample from the support

(4) Immediately flush the sample under deionized water for at least 1 minute

(5) Dry sample with $\mathrm{N}_{2}$ or air. 

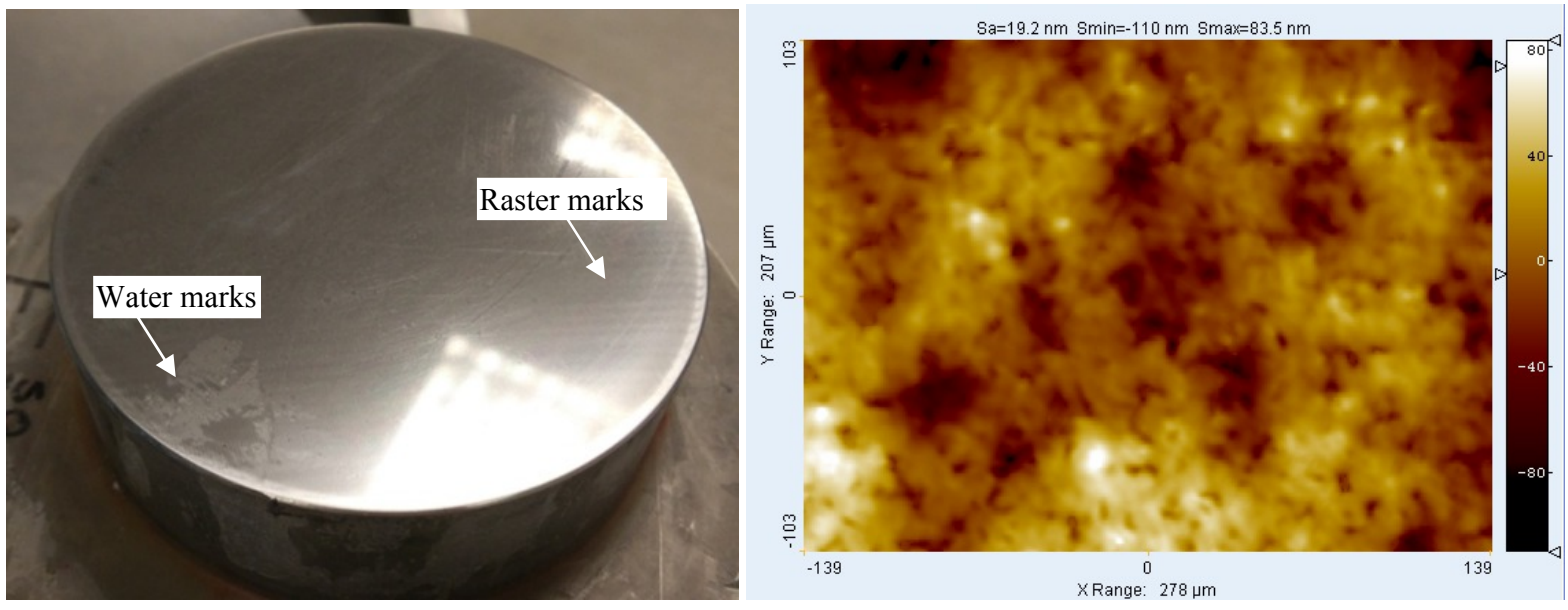

Figure 2. Surface quality and texture measurement $(\mathrm{Sa}=19.2 \mathrm{~nm})$ after Polyurethane pad with Logitech slurry

After 14 minutes polishing, the surface quality was improved, achieving Sa $=6.29 \mathrm{~nm}$ as measured by white light interferometer. The results are shown in Figure 3.

Table 2 Process parameters using Uninap cloth

\begin{tabular}{|c|c|c|c|}
\hline Polishing Cloth & Uninap & Tool Overhang $(\mathrm{mm})$ & 0 \\
\hline Slurry & Logitech & Tool Pressure $(\mathrm{bar})$ & 0.5 \\
\hline Process angle (degree) & 15 & Track Spacing $(\mathrm{mm})$ & 1 \\
\hline Head Speed $(\mathrm{rpm})$ & 1000 & Surface Feed $(\mathrm{mm} / \mathrm{min})$ & 800 \\
\hline Tool Offset $(\mathrm{mm})$ & 0.6 & Removal rate $\left(\mathrm{mm}^{3} / \mathrm{min}\right)$ & 0.483 \\
\hline
\end{tabular}
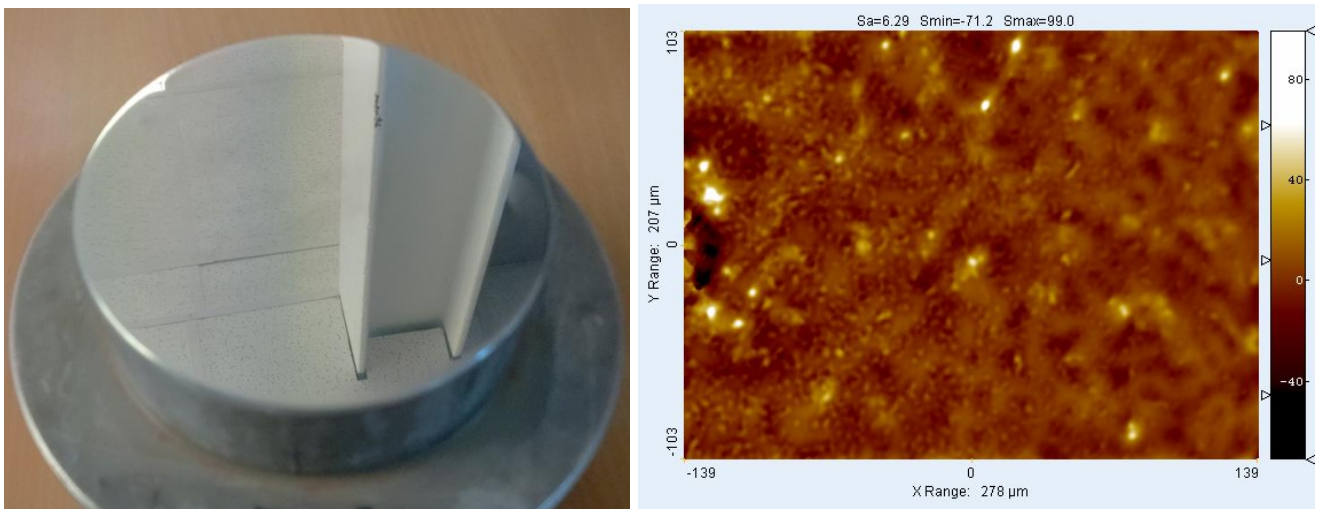

Figure 3. Surface quality and texture measurement $(\mathrm{Sa}=6.29 \mathrm{~nm})$ after Uninap pad with Logitech slurry

\subsection{Non-crystallizing colloidal silica solution}

Logitech CMP solution was designed for lapping applications where the part is face-down on a significantly over-sized lapping plate. This is a configuration exhibiting a large contact-area, which both reduces crystallization, and speeds the process. With the substantially sub-diameter polishing spots of Zeeko Precessions ${ }^{\mathrm{TM}}$, as required for aspheric and freeform surfaces, the Logitech CMP solution proved too sensitive to water-marks and scratching, from slurrycrystallization and oxidization of the aluminum. Especially for polishing a large, face-up part, our experience was that it was not possible to maintain consistent slurry conditions at the tool-part interface over the required polishing runs measured in hours. 
This was demonstrated by polishing a 490mm diameter, off-axis Cassegrain primary mirror in 6082 aluminum alloy. This was for a Phase A study for a proposed infrared space mission. The mirror was first CNC milled at OTI Ltd to lightweight the rear, and generate the front off-axis parabolic surface, and then diamond-turned at the University of Durham facility. We then processed the part on the IRP1200 CNC polishing machine at OpTIC, using bonnet tooling with a polyurethane pad. The abrasive was the standard re-circulated cerium oxide, not normally the first choice for aluminum, but which conveniently delivered a uniform grey surface with attenuated diamond-turning marks. Then, the part was polished on the IRP1200 using re-circulated Logitech SF1 slurry and a Uninap pad. The polishing parameters were as per Table 2. After 4 hours polishing, the marks shown in Figure 4 were produced by hard crystallized particles on the surface of the part.

Following this, three grades of non-crystallizing colloidal silica slurries were investigated. These were acidic colloidal alumina with a $\mathrm{pH}$ between 3 and 3.5 , which provided a chemical and mechanical polishing action. The grades corresponded to fine, intermediate and coarse, corresponding to slow, medium and fast removal rates, respectively. The removal rates and finishing textures were tested on a 100 diameter concave witness part. The test results are tabulated below, in Table 3, and the finished surface of the 490 diameter mirror is shown in Figure 5.

Following these results, we adopted a two-stage process for subsequent work, omitting the intermediate slurry.

Table 3 The removal rate and texture test

\begin{tabular}{|c|c|c|c|}
\hline Slurry & Pad type & Vol. rem. rate $\mathrm{mm}^{3} / \mathrm{min}$ & Texture \\
\hline Colloidal silica coarse & Polyurethane & 16.5 & Grey \\
\hline Colloidal silica intermediate & Uninap & 0.87 & Grey \\
\hline Colloidal silica fine & Uninap & 0.84 & Specular, Sa $\sim 6 \mathrm{~nm}$ \\
\hline
\end{tabular}

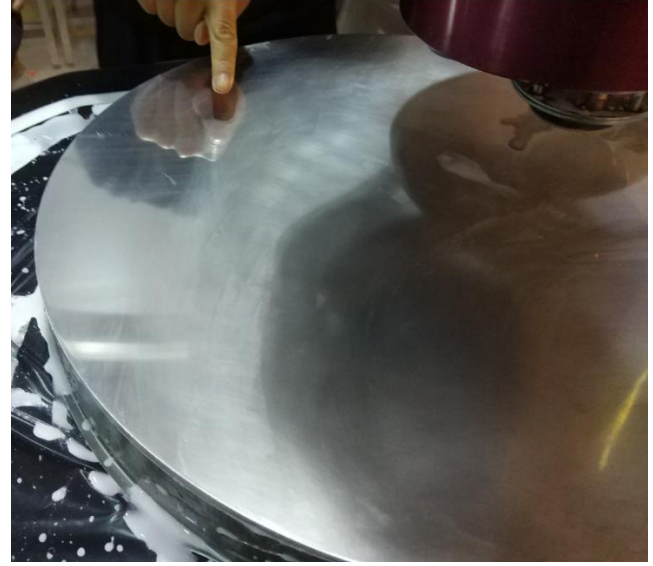

Figure 4. Surface of $490 \mathrm{~mm}$ diameter aluminum part after 4 hours Logitech SF1 slurry polishing

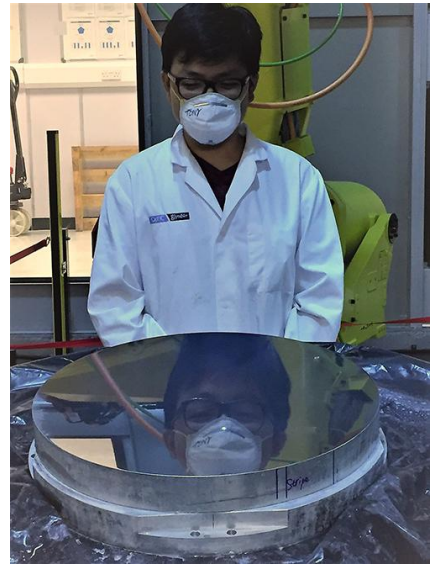

Figure 5. Finished surface of $490 \mathrm{~mm}$ diameter aluminum part after noncrystallizing colloidal silica slurry

\section{FREEFORM ALUMINUM BENDING RIG AND NON-NEWTONIAN TOOL}

We investigated three specialized non-crystallizing colloidal silica slurries including the removal rate and texture with different polishing cloth, and focused on removal of mid-spatial frequency errors on aspherical aluminum surface. A non-Newtonian material tool driven by a randomized unicursal tool path was designed and implemented on an industrial robot (detailed in following section). To demonstrate the whole process chain, a bending rig was designed to create a $400 \mathrm{~mm}$ square free-form surface. 


\subsection{Bending rig to create a free-form surface}

A free-form signature was created by deforming a $3 \mathrm{~mm}$ thick, flat, aluminum plate, grade $1050 \mathrm{~A}$, known for its good corrosion resistance, high ductility and highly reflective finish. Material properties are shown in table 4 .

Table 4. Some specific material properties of Alloy 2015A

\begin{tabular}{|c|c|c|c|c|c|c|c|}
\hline Properties & $\begin{array}{c}\text { Density } \\
\left(\mathrm{g} / \mathrm{cm}^{3}\right)\end{array}$ & $\begin{array}{c}\text { Modulus of } \\
\text { Elasticity } \\
(\mathrm{GPa})\end{array}$ & $\begin{array}{c}\text { Thermal } \\
\text { Expansion }(/ \mathrm{K})\end{array}$ & $\begin{array}{c}\text { Proof } \\
\text { Stress } \\
(\mathrm{MPa})\end{array}$ & $\begin{array}{c}\text { Tensile } \\
\text { Strength } \\
(\mathrm{MPa})\end{array}$ & $\begin{array}{c}\text { Hardness } \\
\text { Brinell } \\
(\mathrm{HB})\end{array}$ & $\begin{array}{c}\text { Melting } \\
\text { point } \\
\left({ }^{\circ} \mathrm{C}\right)\end{array}$ \\
\hline Alloy 2015A & 2.71 & 71 & $24 \times 10^{-6}$ & 85 & $105-145$ & 34 & 650 \\
\hline
\end{tabular}

The original purpose of this aluminum plate was to support a $3 \mathrm{~mm}$ thick sheet of plate glass cemented on top, used to investigate freeform glass polishing [9]. In these new experiments, the glass was removed and the bare aluminum used. The schematic setup is shown in Figure 6. The aluminum bending-plate was mounted spanning two stainless steel support-rails. The deformation was applied by an adjustable screw pulling down on a metal pull-bar attached across the center of the plate. In order for the deformed surface to resist polishing forces with negligible additional deformation, ISOPONTM P38 car-body filler was inserted to fill the gap under the bending-plate, with thin isolating plastic sheets above and below to prevent adhesion. After the $\sim 30$ min curing time for ISOPONTM P38 it's hard, facilitating rapid turnaround of experiments. After processing the deformed part, it was returned to the unstressed, nominally-flat state, to facilitate surface measurement and interpretation. Further details of the procedure are described in [9]. The deformation of the surface was measured by a portable coordinate measuring arm HEXAGON with uncertainty of measurement better than $10 \mu \mathrm{m}$. Figure 7 shows the strong saddle shape surface with PV 1.3mm achieved. This has proved ideal for investigating effects of tool miss-fit, MSFs etc.

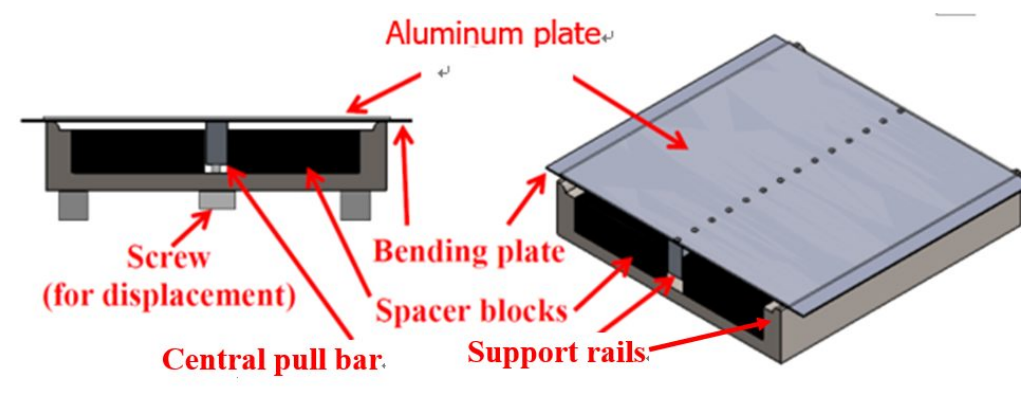

Figure 6. The schemtic aluminum bending rig

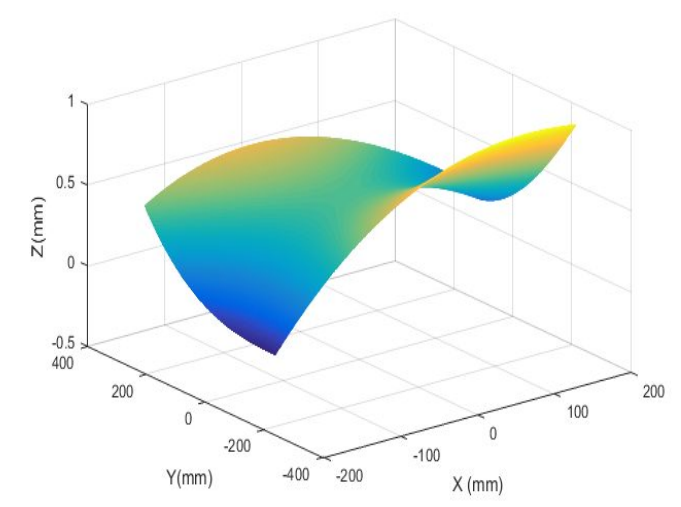

Figure 7. A surface with a strong saddle shape surface with $P V \sim 1.3 \mathrm{~mm}$

\subsection{Non-Newtonian material tool design}

For processing a significantly aspheric or freeform surface, a compliant tool is needed to conform to the local surface contours of the part, but which can then contradict the ability to smooth MSFs. In order to resolve this, a non-Newtonian material (Silly Putty ${ }^{\mathrm{TM}}$ ) in the tool was used, building on the work of the University of Arizona Mirror Lab [11] 
polishing $8.4 \mathrm{~m}$ off-axis paraboloids. Unlike U-of-A, we have also used such a tool in rotation to increase volumetric removal rate, the upper-limit being set by the transition of the Silly Putty ${ }^{\mathrm{TM}}$ from its $\mathrm{n}-\mathrm{N}$ behavior to fully-hard. The spindle speed is optimized by dynamic FEA to balance the tool mis-match and rigidity. This simulation is descried in the next section, based on a tool with $\sim 125 \mathrm{~g}$ of Silly Putty ${ }^{\mathrm{TM}}$, some $10 \mathrm{~mm}$ thick, sandwiched between a rigid back plate and a flexible Bellofram ${ }^{\mathrm{TM}}$ diaphragm, to which the polishing pad (100 $\mathrm{mm}$ in diameter) was cemented - see figure 8 .

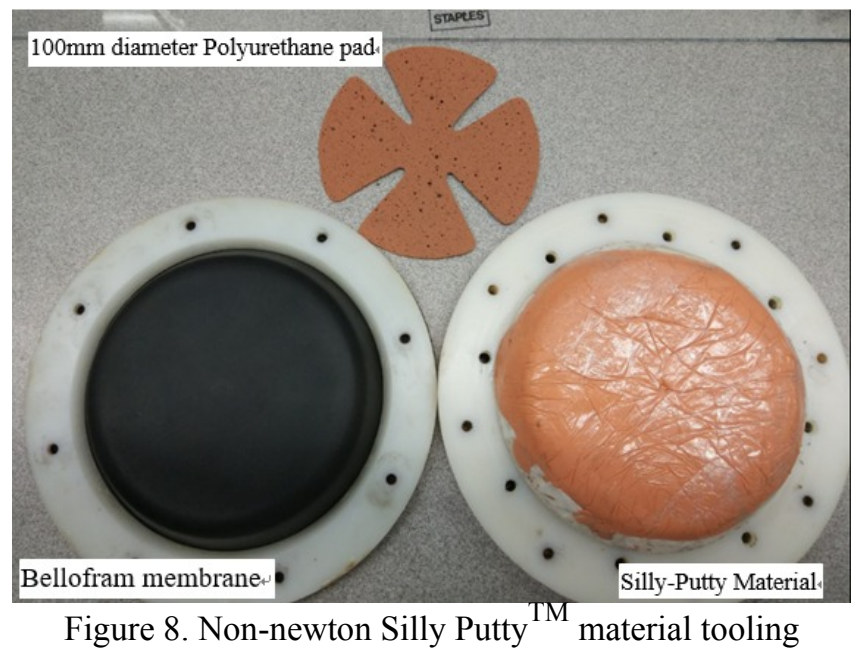

\subsection{Non-newtonian material tool FEA simulation}

The FEA simulation was conducted using ANSYS13.0. Figure 9 shows the CAD mesh model. In this simulation, the polishing force was applied by the $10 \mathrm{Kg}$ self-weight of the tool. The pressure distribution exerted on the surface of the part was calculated. The simulation results with different spindle speeds are shown in Figure 10. It can be seen that the pressure on the part became non-uniform when spindle speed increased from $33 \mathrm{rpm}$ to 300rpm, where the tool also became rigid. Using Preston's Law, the removal function for each spindle speed was calculated. The more rigid the tool becomes, the more capable it is to remove input-MSFs, but local high pressure variations tend to produce new MSFs. At lower spindle speeds, the non-Newtonian material has sufficient time to deform conforming to the local surface of the part, while at higher speeds, it was too stiff.

As the tool is rotationally-symmetric, and rotating in the dynamic FEA model, there should be no azimuthal directionality in the pressure distribution. Therefore, the localized low-pressure regions in Figs $10 \mathrm{~A}$ and $\mathrm{B}$ are believed to be due to i) the meshing pattern as per Figure 9, and ii) the discretization of the colour look-up table in the ANSYS displayed data. The 300rpm option (Figure $10 \mathrm{~B}$ ) was attractive in terms of high removal rate. However, it was rejected due to dynamic instability in use, and the fifteen times radial variation in predicted pressure. Therefore, the 100 rpm option was adopted, which was stable in operation, more uniform in pressure distribution, and delivered a higher removal rate than the 33rpm option.

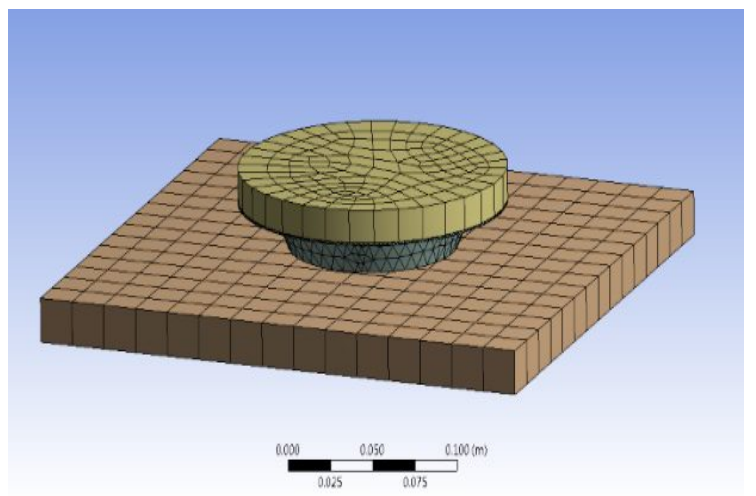

Figure 9. Silly Putty ${ }^{\mathrm{TM}}$ tool CAD mesh model 


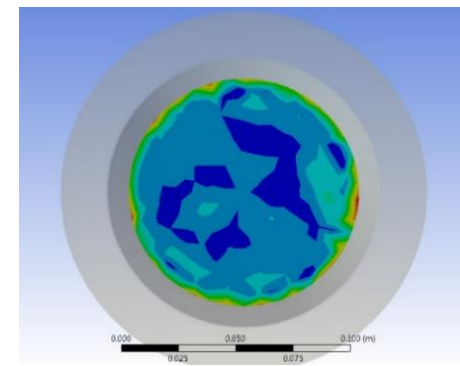

(a) $33 \mathrm{rpm}$

Scale: $8508-83185 \mathrm{~Pa}$

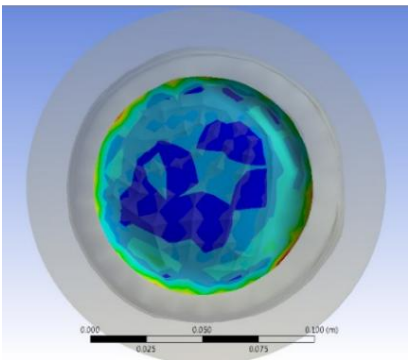

(b) $100 \mathrm{rpm}$

Scale: $7472-84349 \mathrm{~Pa}$

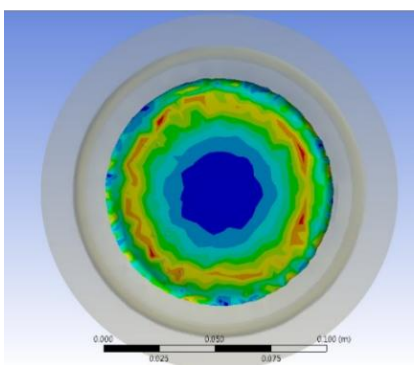

(c) $300 \mathrm{rpm}$

Figure 10. Pressure distribution EFA results (autoscaled) with different rotate speeds

\section{EXPERIMENTS AND RESULTS}

\subsection{Surface preparation of the un-stressed plate by grolishing}

The $3 \mathrm{~mm}$ thick flat aluminum plate, grade 1050A was supplied hardened by rolling, and not annealed. The experiments were conducted on the $400 \mathrm{~mm} \times 400 \mathrm{~mm}$ square bending rig. It was observed that there were many scratches and some deep grooves, attributed to the previous use supporting - and detaching - glass plates. Consequently, it was necessary to clean-up the surface and remove these defects before further work. This was performed on the plate in its nominally-flat, unstressed state, using grolishing on an industrial robot. Two runs were conducted, in orthogonal raster directions. The first used a $100 \mathrm{~mm}$ diameter brass pad with $20 \mu \mathrm{m} \mathrm{AI}_{2} \mathrm{O}_{3}$ abrasive. The second finished the surface with a $100 \mathrm{~mm}$ diameter polyurethane pad on a Neoprene base, with $9 \mu \mathrm{m} \mathrm{AI}_{2} \mathrm{O}_{3}$ abrasive to smooth the surface. The grolishing tools are shown in Figure 10a. After the total process time of 4 hours, the final nominally-flat grolished surface was grey, with $5 \mathrm{~mm}$ periodicity raster-features, as shown in Figure 11b. With the Swinging Part Profilometer (SPP) [16], the total removal depth of $20 \mu \mathrm{m}$ was measured. The depth of the periodic raster features was measured with a Talysurf Intra portable profilometer, and varied between $\sim 0.5 \mu \mathrm{m}$ and $\sim 1.5 \mu \mathrm{m}$ (Figure 12).

Next, the plate was deformed using the adjusting screw. These provided the MSF signatures for the next stage of the experiment, the objective of which was to remove these features. The details are described in next section.

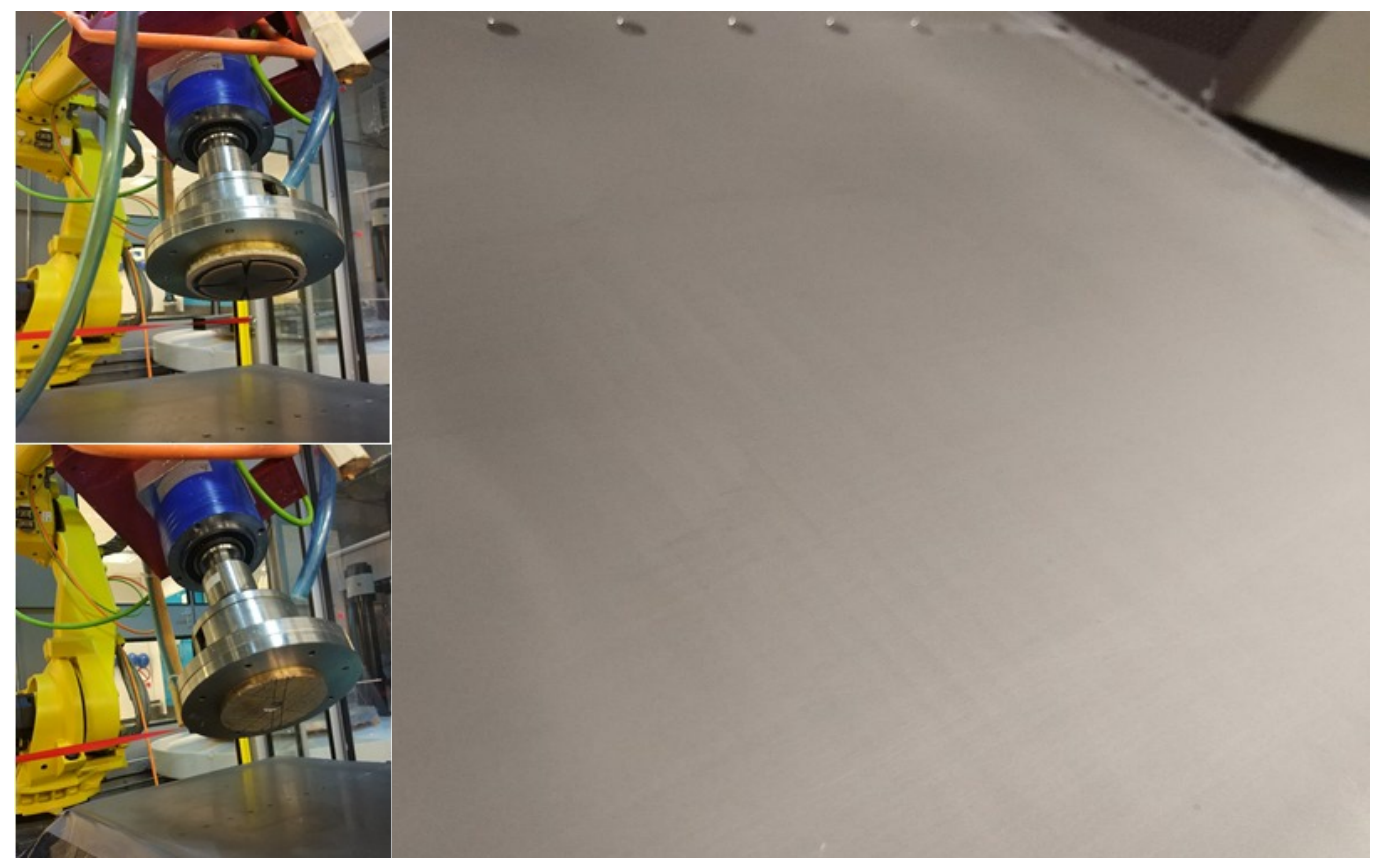

Figure. 11a (left) The robotic $100 \mathrm{~mm}$ diameter grolishing tools - above is the polyurethane pad on a Neoprene base, below the brass tool. Figure $11 \mathrm{~b}$ (right) is the resulting un-deformed, grolished, aluminum surface 

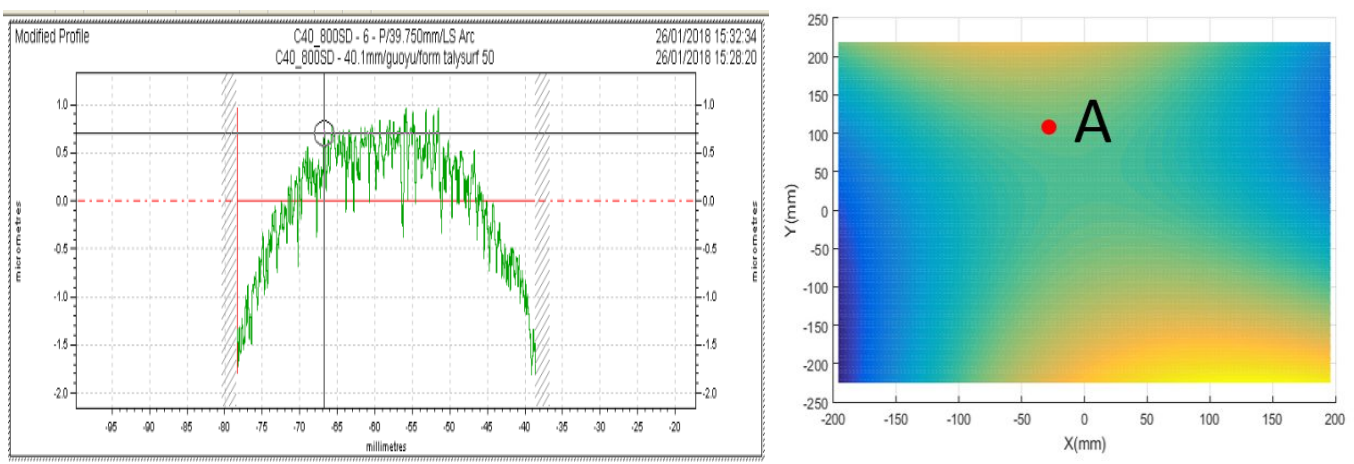

(a) MFS measurement at location A on nominally-flat surface
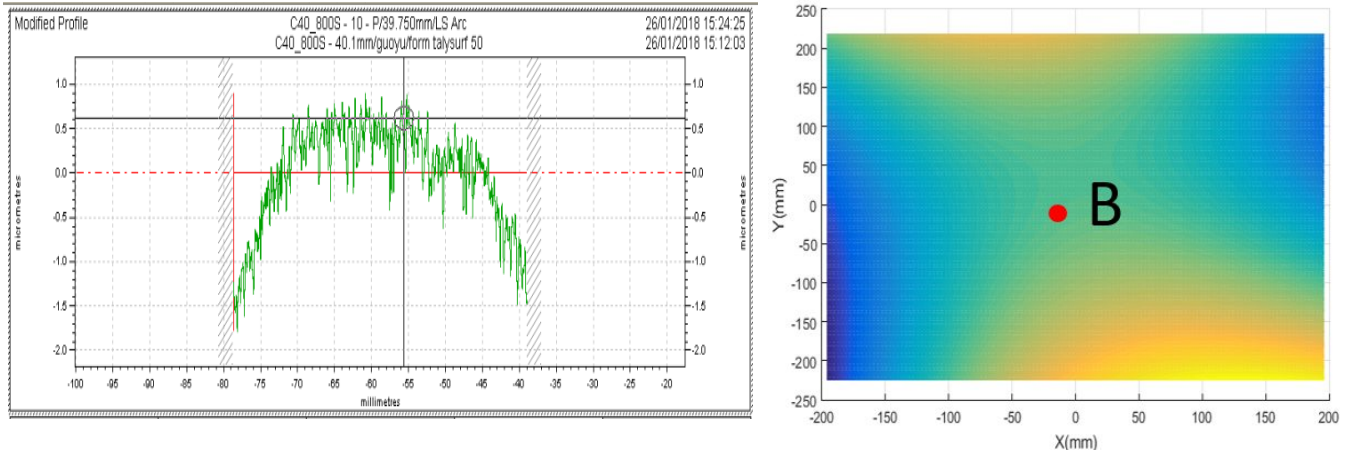

(b) MFS measurement at location B on nominally-flat surface
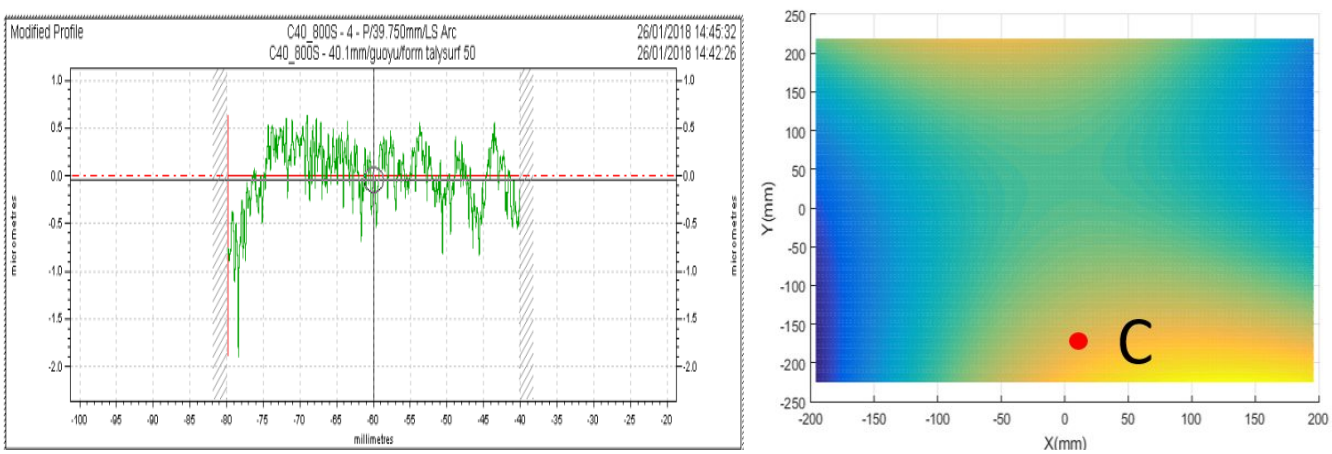

(c) MFS measurement at location C on nominally-flat surface

Figure 12. MSFs measurements by Talysurf Intra Profilometer after hard tool prolishing

\subsection{MSF removal on the free-form stressed plate}

\subsubsection{Robotic polishing set-up and tool path generation}

As shown in Figure 13, a $100 \mathrm{~mm}$ diameter non-Newtonian Silly Putty ${ }^{\mathrm{TM}}$ tool was implemented onto a FANUC R$2000 \mathrm{iB}$ industrial robot. This is currently located at the National Facility for Ultra Precision Surface at OpTIC, by courtesy of Zeeko Ltd. It has $3.05 \mathrm{~m}$ reach and $125 \mathrm{Kg}$ maximum-payload. An ABB motorized spindle with detachable gearbox is mounted as an end-effector to the robot arm, enabling tool-rotation with options of high-speed, low torque, or, high-torque, low-speed. Tooling 'floats' in $\mathrm{Z}$ on the surface of the part under its self-weight, and is driven in the X-Y plane by a gimbal-type coupling. The weight of the tool was $10 \mathrm{~kg}$.

The polishing pads were grooved before cementing to the tool, as shown in Figure 13 on the right. This allowed the slurry adequately flows into the polishing area. The slurry was delivered by a digitally-controlled peristaltic pump with accuracy of $0.1 \mathrm{ml} / \mathrm{mins}$. 
MATLAB functions were added to Zeeko-TPG to allow for the testing of generalized tool paths. These functions take the Cartesian coordinates as generated by Zeeko-TPG and format them correctly for the robot. For the Fanuc robot, the tool path is output as an LS file; a text file which is converted to a binary TP file readable by the robot. LS files consist of definitions of positions and motions. The position definitions are given in six axes: $x, y, z, w, p$, and $r$. The angles $w, p$, and $r$ are computed with respect to the surface normal of the workpiece, which is obtained from Zeeko-TPG. The position definition also includes information about the frame-of-reference of the coordinates [10].

A randomized unicursal, which can be configured to give different randomized paths with almost identical input parameters was trialed in this polishing process chain. This tool path was recently developed and implemented into Zeeko-TPG. The results shown that a non-Newtonian tool following this tool path can smooth surfaces and effectively remove MSFs, as exemplified in in the next section. The mathematical basis of this tool path development will be published in separated paper.
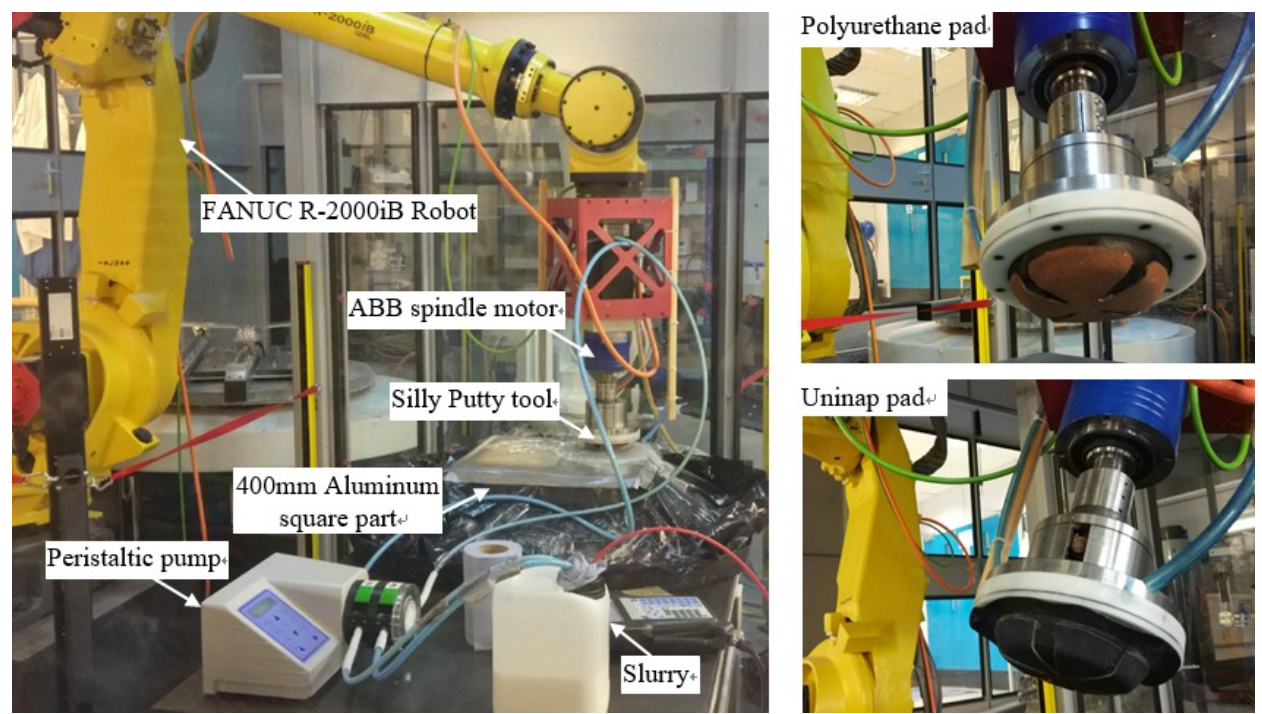

Figure 13. Robotic polishing using non-Newtonian tool

\subsubsection{Experiment and results on the free-form stressed plate}

Following Section 2.2, a two-step polishing process was adopted, as shown in Table 4, to attempt removal of the MSF raster-features created in the previous hard-tool grolishing (Section 4.1).

Table 4. The parameters for the process chain

\begin{tabular}{|l|c|c|c|c|c|c|c|}
\hline & $\begin{array}{c}\text { Tool } \\
\text { Material }\end{array}$ & Slurry & $\begin{array}{c}\text { Polishing } \\
\text { pad }\end{array}$ & $\begin{array}{c}\text { Tool Rotation } \\
(\mathrm{rpm})\end{array}$ & Tool path & $\begin{array}{c}\text { Polishing } \\
\text { force (kg) }\end{array}$ & $\begin{array}{c}\text { Polishing time } \\
\text { (minutes) }\end{array}$ \\
\hline Step 1 & Silly Putty ${ }^{\mathrm{TM}}$ & $\begin{array}{c}\text { Coarse } \\
\text { slurry }\end{array}$ & Polyurethane & 100 & $\begin{array}{c}\text { Randomized } \\
\text { unicursal tool } \\
\text { path }\end{array}$ & 10 & 168 \\
\hline Step 2 & Silly Putty & $\begin{array}{c}\text { FM } \\
\text { slurry }\end{array}$ & Uninap & 100 & $\begin{array}{c}\text { Randomized } \\
\text { unicursal tool } \\
\text { path }\end{array}$ & 10 & 84 \\
\hline
\end{tabular}

After both steps, the total removal depth of $16 \mu \mathrm{m}$ was calculated from the measured volumetric removal rate. The part was then relaxed to its un-stressed nominally-flat condition for measurement. Three Intra profilometer scans were conducted to identify the depth of MFSs at the same locations A, B and C in Figure 12. The measurements are shown in Figure 14. It can be seen that that the input $\sim 0.5 \mu \mathrm{m}$ to $\sim 1.5 \mu \mathrm{m}$ deep MSFs (shown in Figure 12) have been effectively removed. The surface quality (shown in Figure 15) was then measured under white-light interferometer. The texture of $\mathrm{Sa} \sim 5 \mathrm{~nm}$ was achieved, as shown in Figure 16, after removing the low order Zernike terms. 


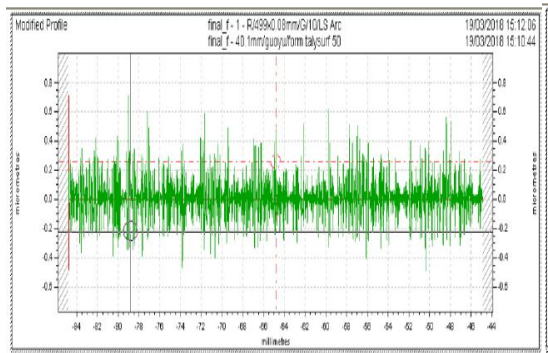

(a) Measurement at location $\mathrm{A}$

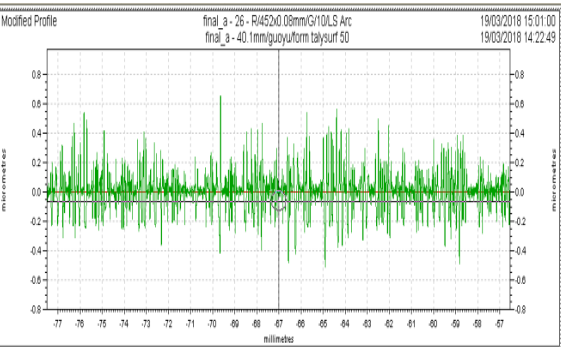

(b) Measurement at location B

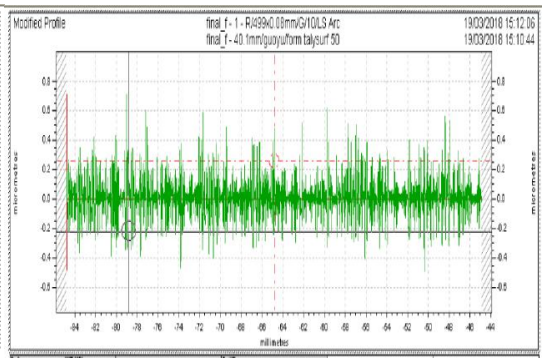

(c) Measurement at location $\mathrm{C}$

Figure 14. MSF measurements by Talysurf Intra Profilometer after processing

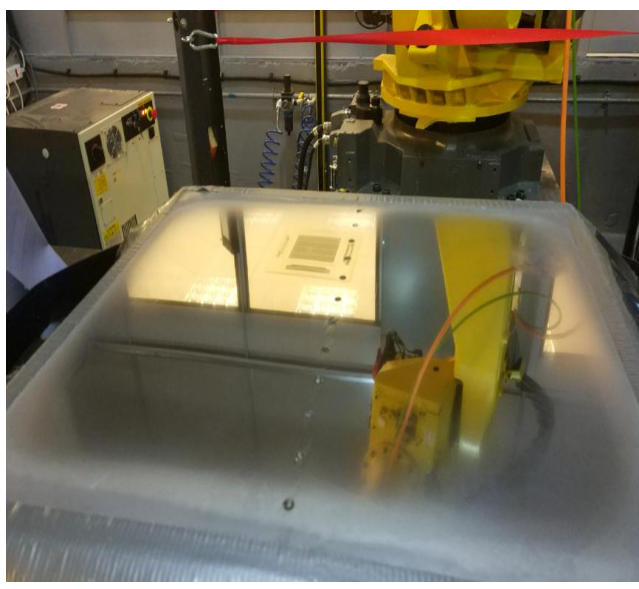

Figure 15. General surface quality after processing

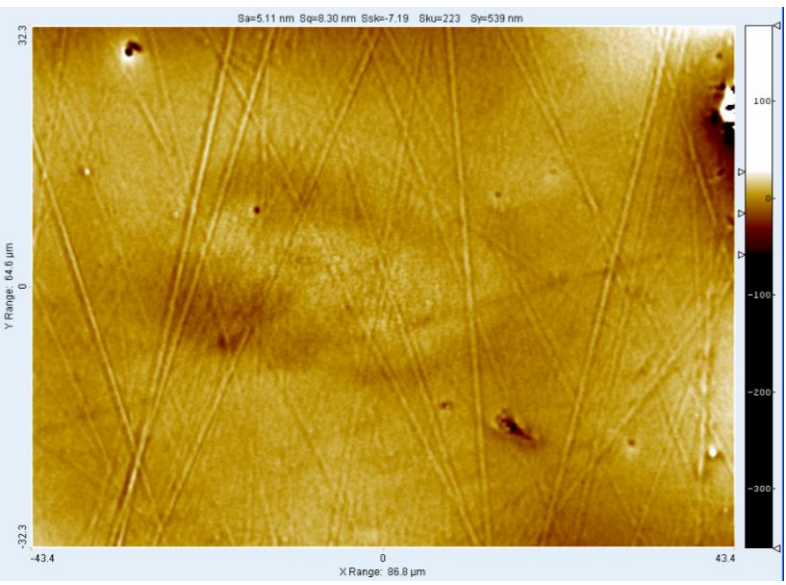

Figure 16. White light interferometer micrograph of texture showing $\mathrm{Sa} \sim 5 \mathrm{~nm}$

\section{CONCLUSION}

Aluminum mirrors become increasingly attractive materials in space and ground-based optical systems due to high specific stiffness, ruggedness, suitability for extreme light-weighting, and thermal conductivity. Conventional SPDT can produce such surfaces, but with diamond turning features which cause deleterious diffraction effects and stray light. To remove the MSFs and improve surface texture, post-polishing may be adopted. However, aluminum is difficult to polish, limited by the material and slurry properties.

In this paper, we focused on the development of an effective process chain, which is able to address these issues for aluminum polishing, especially for large aspherics and free-forms. This includes removal of MSF errors, texture control and automation of manual interventions to accelerate the whole process. We have investigated different aluminum polishing slurries from different suppliers and identified two effective slurries and pads, as steps in the process chain. To achieve fast removal rate and control of surface texture, we designed a non-Newtonian tool, which follows a speciallydeveloped random tool-path. The whole process-chain has been demonstrated on the $400 \mathrm{~mm}$ square free-form surface on an industrial robot. The MSFs from hard-tool grolishing have been removed, and texture of $\mathrm{Sa} \sim 5 \mathrm{~nm}$ achieved. This also demonstrated the ability to automate some aspects of the whole process for mass-production of aluminum mirrors.

\section{REFERENCES}

[1] K. J. Moeggenborg, C. Barros, S. Lesiak, N. Naguib, and S. Reggie, "Low-scatter bare aluminum optics via chemical mechanical polishing," Current Developments in Lens Design and Optical Engineering Ix, vol. 7060, 706002(2008). 
[2] K. G. Carrigan, "Visible Quality Aluminum and Nickel Superpolish Polishing Technology Enabling New Missions," Infrared Technology and Applications Xxxvii, vol. 8012, 80123f(2011).

[3] D. Vukobratovich and J. P. Schaefer, "Large Stable Aluminum Optics for Aerospace Applications," Optomechanics 2011: Innovations and Solutions, vol. 8125, 81250t(2011).

[4] M. Gubarev, J. K. Kolodziejczak, C. Griffith, J. Roche, W. S. Smith, T. Kester, et al., "Development of a direct fabrication technique for full-shell x-ray optics," Space Telescopes and Instrumentation 2016: Ultraviolet to Gamma Ray, vol. 9905, 99051v(2016).

[5] K. G. Carrigan, "Manufacturing Status of Tinsley Visible Quality Bare Aluminum and an Example of Snap Together Assembly," Infrared Technology and Applications Xxxviii, Pts 1 and 2, vol. 8353, 83532d(2012).

[6] S. L. Folkman and M. S. Stevens, "Characterization of electroless nickel plating on aluminum mirrors," Optomechanical Design and Engineering 2002, vol. 4771, 254-264(2002).

[7] J. Kinast, E. Hilpert, R. R. Rohloff, A. Gebhardt, and A. Tunnermann, "Thermal expansion coefficient analyses of electroless nickel with varying phosphorous concentrations," Surface \& Coatings Technology, vol. 259, 500503(Nov 25 2014).

[8] T. Newswander, B. Crowther, G. Gubbels, and R. Senden, "Aluminum alloy AA-6061 and RSA-6061 heat treatment for large mirror applications," Material Technologies and Applications to Optics, Structures, Components, and Sub-Systems, vol. 8837, 883704(2013).

[9] D. Walker, W. Hsing-Yu, G. Y. Yu, H. Y. Li, W. Zhang, and C. L. Lu, "Insight into aspheric misfit with hard tools: mapping the island of low mid-spatial frequencies," Applied Optics, vol. 56, 9925-9931(Dec 20 2017).

[10] D. Walker, C. Dunn, G. Y. Yu, M. Bibby, X. Zheng, H. Y. Wu, et al., "The role of robotics in computer controlled polishing of large and small optics," Optical Manufacturing and Testing Xi, vol. 9575, 95750b(2015).

[11] D. W. Kim and J. H. Burge, "Rigid conformal polishing tool using non-linear visco-elastic effect," Optics Express, vol. 18, 2242-2257(Feb 1 2010).

[12] M. F. Dasilva, K. Shimizu, K. Kobayashi, P. Skeldon, G. E. Thompson, and G. C. Wood, "On the Nature of the Mechanically Polished Aluminum Surface," Corrosion Science, vol. 37, 1511-1514(Sep 1995).

[13] R. ter Horst, N. Tromp, M. de Haan, R. Navarro, L. Venema, and J. Pragt, "Directly Polished Light Weight Aluminum Mirror," Advanced Optical and Mechanical Technologies in Telescopes and Instrumentation, Pts 13, vol. 7018, 701808(2008).

[14] D. D. Walker, R. Freeman, R. Morton, G. McCavana, and A. Beaucamp, "Use of the 'Precessions'(TM) process for prepolishing and correcting 2D \& 2 1/2 D form," Optics Express, vol. 14, 11787-11795(Nov 27 2006).

[15] D. D. Walker, A. T. H. Beaucamp, D. Brooks, V. Doubrovski, M. Cassie, C. Dunn, et al., "Recent development of Precessions polishing for larger components and free-form surfaces," Current Developments in Lens Design and Optical Engineering $V$, vol. 5523, 281-289(2004).

[16] P. Zhang, J. Li, G. Y. Yu, and D. D. Walker, "Development of Swinging Part Profilometer for Optics," Optics and Measurement International Conference 2016, vol. 10151, 101510b(2016). 